\title{
The impact of a produce prescription programme on healthy food purchasing and diabetes-related health outcomes
}

\author{
Julian Xie ${ }^{1,2, *}$ (D), Ashley Price ${ }^{2}, \mathrm{Neal}_{\text {Curran }}{ }^{2}$ and Truls $\varnothing_{\text {stbye }}{ }^{2}$ \\ 'Duke University, School of Medicine, Durham, NC, USA: ${ }^{2}$ Duke University, Department of Family Medicine and \\ Community Health, Suite 400, 2200 W. Main Street, Durham, NC 27705, USA: ${ }^{3}$ Reinvestment Partners, Durham, \\ NC, USA
}

Submitted 27 September 2020: Final revision received 10 April 2021: Accepted 22 April 2021: First published online 27 April 2021

\begin{abstract}
Objective: To evaluate a Produce Prescription Programme's utilisation and its effects on healthy food purchasing and diabetes control among participants with type 2 diabetes.

Design: Prospective cohort study using participants' electronic health records and food transaction data. Participants were categorised as 'Frequent Spenders' and 'Sometimes Spenders' based on utilisation frequency. Multivariate regressions assessed utilisation predictors and programme effects on fruit/vegetable purchasing (spending, expenditure share and variety) and on diabetes-related outcomes (HbA1c, BMI and blood pressure).

Setting: Patients enrolled by clinics in Durham, North Carolina, USA. Participants received $\$ 40$ monthly for fruits and vegetables at a grocery store chain.

Participants: A total of 699 food-insecure participants (353 with diabetes).

Results: Being female and older was associated with higher programme utilisation; hospitalisations were negatively associated with programme utilisation. Frequent Spender status was associated with $\$ 8.77$ more in fruit/vegetable spending $(P<0.001), 3.3 \%$ increase in expenditure share $(P=0.007)$ and variety increase of 2.52 fruits and vegetables $(P<0.001)$. For $\$ 10$ of Produce Prescription Dollars spent, there was an $\$ 8.00$ increase in fruit/vegetable spending $(P<0.001), 4.1 \%$ increase in expenditure share and variety increase of 2.3 fruits/vegetables $(P<0.001)$. For the 353 participants with diabetes, there were no statistically significant relationships between programme utilisation and diabetes control.

Conclusions: Programme utilisation was associated with healthier food purchasing, but the relatively short study period and modest intervention prevent making conclusions about health outcomes. Produce Prescription Programmes can increase healthy food purchasing among food-insecure people, which may improve chronic disease care.
\end{abstract}

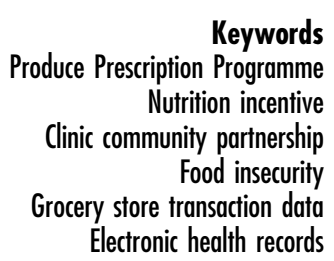

Equitable access to healthy food remains an unmet need in North Carolina, as in much of the United States. Structural inequities create food environments with few healthy options and abundant processed food and fast food ${ }^{(1)}$. North Carolina is consistently one of the most foodinsecure states in the country, ranking tenth highest in $2018^{(2,3)}$. In Durham, $13 \cdot 5 \%$ of Durham residents were food insecure and one-third of adults had obesity in 2018, and
'Obesity, Diabetes, and Food Access' is among residents' top health priorities ${ }^{(4)}$.

The Supplemental Nutrition Assistance Program (SNAP) helps low-income households meet basic food needs. However, SNAP beneficiaries often have lower diet quality because of economic pressure to stretch benefits by purchasing energy-dense but nutrient-poor foods ${ }^{(5)}$. Thus, food insecurity can cause and exacerbate chronic disease 
despite existing food assistance resources ${ }^{(6)}$. Nutrition incentive programmes attempt to address this problem by subsidising healthy food purchases. Thus, nutrition incentive programmes increase purchasing power for food and promote healthy food intake among lowsocio-economic status individuals ${ }^{(7)}$.

To further build upon nutrition incentive programmes, community-based organisations and health centres have invested in Produce Prescription Programmes. In these programmes, healthcare workers identify food-insecure patients and 'prescribe' monetary incentives to purchase fruits and vegetables, or healthy food provided onsite in health settings ${ }^{(8)}$. These innovative programmes incorporate food access directly into the patient-provider relationship, with the intent of promoting healthy eating by better-enabling patients to follow healthcare providers' dietary advice. Produce Prescription Programmes are relatively new, reaching prominence in the 2010s through various pilot programmes and new US government funding initiatives under the 2014 and 2018 Farm Bills ${ }^{(9)}$.

To date, Produce Prescription Programme research has often focused on small pilot programmes with limited use of food transaction and health data ${ }^{(9)}$. A scoping review of 186 Food as Medicine interventions found that $72 \%$ of interventions and retail nutrition programmes were effective at achieving one or more of the outcomes reported, with the most effective being multi-component interventions combining price incentives, nutrition incentives and marketing nudges. However, the review points to a notably low number of studies on how Food as Medicine programmes interact with specific disease diagnoses and health outcomes. Only twenty of 186 studies focused on populations with specific diseases; ten of these targeted diabetes ${ }^{(10)}$.

We evaluated a new Durham Produce Prescription Programme facilitated by the non-profit organisation Reinvestment Partners. This programme gave foodinsecure patients $\$ 40$ monthly to spend on fruits and vegetables at a major grocery store chain. Our study is novel in its integration of food transaction data and electronic health records (EHR). The analytic sample included participants whose food transaction data could be matched to their EHR. Our study aimed to (1) assess predictors of programme utilisation; (2) assess the association between programme utilisation and healthy food purchasing and (3) explore the association between programme utilisation and diabetes control among the participant subsample with type 2 diabetes.

\section{Methods}

\section{The intervention}

Healthcare workers identified food insecure patients, using screening tools such as the Hunger Vital Sign or their informal understanding of patients' socio-economic situations, and enrolled them into the Produce Prescription Programme using an online portal between May 2018 and June $2019^{(11)}$. Patients were eligible if they received SNAP and were over 18 years old. Enrolment required a partner grocery store chain loyalty card; patients without a loyalty card acquired one as part of enrolment. Enrolment sites included a federally qualified health centre, an academic outpatient clinic serving low-income patients and two organisations that refer seniors to health-related social services. Social workers, case managers, dietitians and medical students enrolled patients during clinic visits, as well as through the clinics' group diabetes education classes. Participants received an instructional handout after enrolment.

Once enrolled, participants received $\$ 40$ monthly for up to 1 year to spend at partner grocery store chain locations in Durham. Produce Prescription Dollars could be spent on Women, Infants and Children programme-approved fruits and vegetables. Eligible items included fresh, frozen or canned fruit, vegetables and beans without added salt, sugar or fat. Participants received $\$ 40$ monthly contingent on purchasing at least one item at a partner grocery store using their SNAP Electronic Benefit Transfer card and store loyalty card. Produce Prescription Dollars were applied automatically as the tender (payment method) of first resort for eligible items.

The Produce Prescription Programme evaluated in the current study was funded by the US Department of Agriculture Food Insecurity Nutrition Incentive programme, and the current study was funded by Blue Cross Blue Shield of North Carolina Foundation.

\section{Data sources}

The study sample included all Produce Prescription participants for whom it was possible to match their programme administration data, grocery store food transaction data and electronic health records (EHR) data. The Duke Health Institutional Review Board approved a waiver of consent for analysis of secondary EHR and programme administration data. We acquired grocery store food transaction data from April 2018 to June 2019, or 1 month prior to the first participant enrolment (Fig. 1). We accessed EHR from November 2017 to June 2019, enabling health data analysis from 6 months before enrolment through the end of programme participation.

From programme administration data, we acquired enrolment date and location and loyalty card number. From EHR, we acquired demographic information, including age, sex and race or ethnicity. We also measured healthcare system utilisation and baseline health status through clinic visits and hospitalisations documented in EHR. From food transaction data, we accessed food item barcodes, descriptions, prices and payment methods. Through payment method, we could determine when participants spent Produce Prescription Dollars. Food transaction data were linked to the participant by loyalty card number. 
Produce prescription impact on food purchases

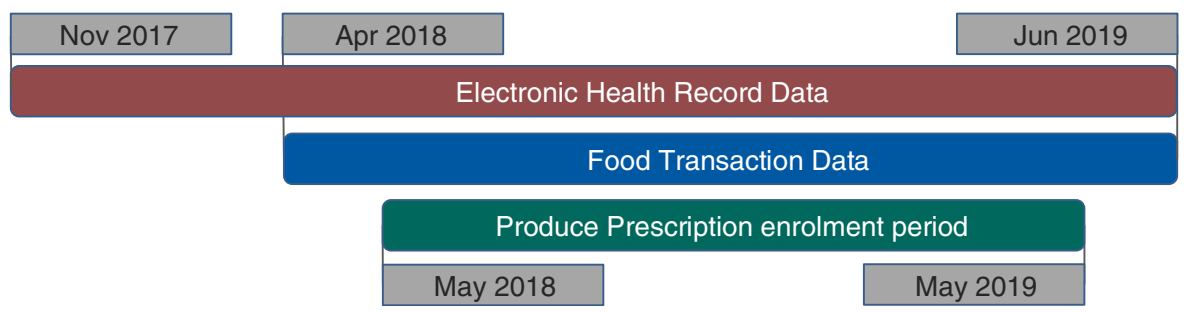

Fig. 1 (colour online) Data timeline for Produce Prescription Programme. Timeline for electronic health records (her), food transaction and Produce Prescription Programme enrolment data. Diagram showing dates of availability for each data source. Red rectangle shows EHR data from November 2017 to June 2019, a blue rectangle shows food transaction data from April 2018 to June 2019 and a green rectangle shows the Produce Prescription enrolment period from May 2018 to May 2019

To merge these data sources, we matched names and phone numbers to medical record numbers, enabling matching of loyalty card numbers to medical record numbers. Using loyalty card numbers and medical record numbers, we merged food transaction data with EHR. We then merged food transaction data with proprietary barcode databases, which enabled food transaction categorisation.

\section{Measures}

To assess predictors of programme utilisation (Aim 1), we measured programme utilisation in two ways. First, we measured it as a binary outcome, where utilisation was defined as having spent Produce Prescription Dollars at least once following enrolment. Second, we calculated a 'utilisation ratio' equal to months in which a participant spent Prescription Dollars divided by months with food transaction data available for that participant.

We used the utilisation ratio to categorise those who used the programme at least once into: (1) Frequent Spenders who either spent Produce Prescription Dollars every month or were in the fourth quartile for utilisation ratio and (2) Sometimes Spenders who spent Prescription Dollars at least once up to the fourth quartile cut-off for utilisation ratio. This categorisation was chosen due to programme utilisation's non-normal distribution and the lack of reliable food transaction data for those who never spent Prescription Dollars after enrolment.

To evaluate effects on healthy food purchasing (Aim 2), we used fruit and vegetable purchasing as the main outcome, and we used three measures similar to those in previous studies ${ }^{(12)}$. First, we used monthly fruit and vegetable spending: dollars spent on fruits or vegetables, including those not eligible for purchase using Produce Prescription Dollars for a more complete approximation of participants' consumption. Second, we calculated monthly fruit and vegetable expenditure share, or fruit and vegetable spending as a proportion of total food spending. Third, we measured fruit and vegetable diversity by counting the unique fruit and vegetable items purchased in a month.

To assess effects on diabetes-related outcomes (Aim 3), we calculated quarter averages for HbA1c (HbA1c), BMI and systolic blood pressure. A participant's first quarter average was calculated using data from a time period 3 months before their individual programme enrolment date. We then calculated averages of the health measures for each quarter thereafter, up to four quarters after enrolment. All quarterly averages were calculated at the individual level, recognising participants could enrol on different dates. We set the first post-enrolment quarter 1 month after enrolment to account for potential lag in programme utilisation and delayed effects on health measures (i.e. accounting for the fact that a participant could enrol within one calendar month but not start spending until the following calendar month).

We also analysed EHR data to acquire demographic and health utilisation measures. We assigned primary health insurance by finding the most commonly listed payor among each participant's healthcare encounters. Health utilisation was measured through outpatient clinic visits, emergency department (ED) visits that did not result in an inpatient admission and hospitalisations in the year following enrolment. If multiple healthcare encounters occurred on the same day, we only counted one of each type at most.

\section{Statistical analysis}

For predictors of programme utilisation (Aim 1), we used $\chi^{2}$ tests and ANOVA to assess whether utilisation differed by demographics, health insurance, enrolment site, baseline health status and healthcare utilisation. We used logistic regression with programme utilisation as a discrete outcome. We also conducted logistic regression with Frequent Spender status as a binary outcome relative to Sometimes Spender status.

To assess how programme utilisation affected healthy food purchasing (Aim 2), we performed multivariable random effects panel regression to assess monthly fruit and vegetable purchasing. For each of the three fruit and vegetable purchasing outcome measures, we tested models with programme utilisation measured by Frequent Spender status or monthly Produce Prescription Dollars spent as the key explanatory variable. For Aim 2, we conducted analyses on the full sample with diabetes diagnosis as one of the covariates. We subsequently also conducted 
analyses on participants with and without diabetes to compare programme effects for these two groups.

To evaluate how programme utilisation affected diabetes-related outcomes (Aim 3), we used multivariable panel regression with 3-month averages of HbA1c, BMI and systolic blood pressure. The key explanatory variables for these models were Frequent Spender status.

For Aims 2 and 3, we included the following potential confounders: demographics (race or ethnicity, sex and age), health insurance, enrolment site, healthcare utilisation (outpatient clinic visits, ED visits and hospitalisations), month-fixed effects and the maximum possible months the participant could have utilised the programme (accounting for potential programme experience differences between early and late participants). For Aim 2, we additionally controlled for non-fruit and vegetable spending as a proxy for household size.

\section{Results}

\section{Subject characteristics}

This Produce Prescription Programme enrolled 807 patients and we matched 699 participants' food transaction data and EHR. Data were not available on how many patients who were offered programme enrolment. The majority of participants were Black (81\%), elderly (52\% were 60 or older) and female ( $72 \%$ ) (Table 1 ).

The average utilisation ratio among those who spent Produce Prescription Dollars at least once was $73 \%$. To distinguish Frequent Spenders from Sometimes Spenders, we excluded people who never utilised the programme and then used the fourth quartile cut-off for utilisation ratio, which was $87.5 \%$. There were 242 Frequent Spenders and 339 Sometimes Spenders. For analyses on healthy food purchasing (Aim 2) and diabetes-related outcomes (Aim 3), we excluded the 119 'Never Spenders' since their spending at our partner grocery stores was so low that their food transaction data were unlikely to represent their consumption accurately.

Further, we conducted a sensitivity analysis including only the 322 participants with food transaction data available before enrolment, meaning they already had a store loyalty card. We expected these individuals' food transaction data to accurately represent their overall food purchase and consumption. Within this sensitivity analysis, the results were similar, so we retained all 581 participants who ever redeemed Produce Prescription Dollars for the analyses in Aim 2 and Aim 3.

\section{Aim 1}

\section{Predictors of programme utilisation}

Table 1 shows the association of demographic characteristics and healthcare utilisation patterns with programme utilisation as a binary outcome, as well as individual $\chi^{2}$ or ANOVA tests for each characteristic. Higher programme utilisation frequency was associated with older age, female sex, Black race, enrolment through senior assistance organisations and fewer ED visits. Participants with Medicare, Medicare Advantage or private insurance were also comparatively more likely to utilise the programme more frequently.

In the multivariate logistic regression also shown in Table 1, higher programme utilisation likelihood was associated with female sex (male sex OR $=0 \cdot 45,95 \%$ CI $0 \cdot 28$, $0 \cdot 73)$, older age (OR $=1.03,95 \%$ CI $1.01,1.05)$ and more outpatient clinic visits $(\mathrm{OR}=1.03,95 \%$ CI 1.00, 1.05). A higher number of hospitalisations (OR $=0.81,95 \% \mathrm{CI}$ $0.75,0.98)$ were associated with lower programme utilisation likelihood.

In an alternative multivariate logistic regression of Frequent Spender $v$. Sometimes Spender status, more frequent utilisation was associated with Medicare $(\mathrm{OR}=2 \cdot 06$, $95 \%$ CI 1.06, 4.31), Medicare Advantage (OR = 1.88, $95 \%$ CI $1.00,3.54)$ and Medicaid (OR $=1.75$, $95 \%$ CI 1.03 , $2 \cdot 98$ ). In both multivariate regressions, programme utilisation was otherwise not significantly associated with other health status and utilisation measures, including diabetes diagnosis, BMI, systolic blood pressure or ED visits.

Table 2 shows a comparison between participants with and without a diabetes diagnosis. Participants with diabetes had a higher average age, which was also evidenced through a higher representation of Medicare enrolees and recruitment from the two senior assistance organisations. Participants with diabetes also had higher baseline HbA1c, BMI and systolic blood pressure and more frequent outpatient clinic visits and hospitalisations.

\section{$\operatorname{Aim} 2$}

Programme influence on bealthy food purchasing Among all Frequent and Sometimes Spenders, fruit and vegetable purchasing increased over time, with Frequent Spenders experiencing the largest growth. Total food spending and fruit and vegetable spending increased the most within the first 2 months of enrolment. Produce Prescription Dollars accounted for the largest share of participants' fruit and vegetable spending growth (Fig. 2). Higher programme utilisation was associated with higher fruit and vegetable purchasing (Table 3 ). In addition, the diabetes diagnosis covariate did not have a statistically significant effect on the full-sample regressions with our three healthy food purchasing outcome measures.

\section{Fruit and vegetable spending}

Frequent Spender status was associated with higher monthly fruit and vegetable spending $(\beta=\$ 8.77$, $P<0.001)$. The effect for participants with diabetes was $\$ 7.51$ compared with $\$ 10.11$ for the sample without a diabetes diagnosis. Each additional Produce Prescription Dollar spent was associated with higher fruit and vegetable 
Table 1 Subject characteristics and predictors of utilisation for a produce prescription programme in Durham, NC, 2018-2019

\begin{tabular}{|c|c|c|c|c|c|c|c|c|c|c|}
\hline \multirow[b]{2}{*}{ Category } & \multicolumn{2}{|c|}{ Overall } & \multicolumn{6}{|c|}{ Distribution by spender type $\neq$} & \multicolumn{2}{|c|}{$\begin{array}{l}\text { Multivariate logistic } \\
\text { regression§ }\end{array}$} \\
\hline & $n$ & $\%$ & \multicolumn{2}{|c|}{$\begin{array}{l}\text { Never } \\
\text { spender }\end{array}$} & \multicolumn{2}{|c|}{$\begin{array}{l}\text { Sometimes } \\
\text { spender }\end{array}$} & \multicolumn{2}{|c|}{$\begin{array}{l}\text { Frequent } \\
\text { spender }\end{array}$} & OR & $95 \% \mathrm{Cl}$ \\
\hline \multirow[t]{2}{*}{ Participants } & \multicolumn{2}{|c|}{699} & \multicolumn{2}{|c|}{118} & \multicolumn{2}{|c|}{339} & \multicolumn{2}{|c|}{242} & & \\
\hline & Mean & SD & Mean & SD & Mean & SD & Mean & SD & & \\
\hline Average age (years) $)^{\star *}$ & $58 \cdot 5$ & $14 \cdot 1$ & $53 \cdot 3$ & $15 \cdot 3$ & $59 \cdot 1$ & 13.7 & $60 \cdot 1$ & $13 \cdot 6$ & 1.03 & $1.01,1.05 \dagger$ \\
\hline \multicolumn{11}{|l|}{$S^{*} x^{\star \star}$} \\
\hline Female & 506 & $72 \cdot 4$ & \multicolumn{2}{|c|}{$13.6 \%$} & \multicolumn{2}{|l|}{$51 \%$} & \multicolumn{2}{|c|}{$35.4 \%$} & $\begin{array}{l}\text { Base } \\
\text { category }\end{array}$ & \\
\hline Male & 193 & $27 \cdot 6$ & \multicolumn{2}{|c|}{$25.4 \%$} & \multicolumn{2}{|l|}{$42 \%$} & \multicolumn{2}{|c|}{$32.6 \%$} & 0.45 & $0.28,0.73 \dagger \dagger$ \\
\hline \multicolumn{11}{|l|}{ Race or ethnicity* } \\
\hline Black & 569 & 81.4 & \multicolumn{2}{|c|}{$15.1 \%$} & \multicolumn{2}{|c|}{$48.7 \%$} & \multicolumn{2}{|c|}{$36 \cdot 2 \%$} & $\begin{array}{l}\text { Base } \\
\text { category }\end{array}$ & \\
\hline White non-Hispanic & 81 & 11.6 & \multicolumn{2}{|c|}{$22.2 \%$} & \multicolumn{2}{|c|}{$43.2 \%$} & \multicolumn{2}{|c|}{$34.6 \%$} & 0.70 & $0.35,1.38$ \\
\hline Hispanic (regardless of race) & 37 & $5 \cdot 3$ & 27 & & $62 \cdot 2$ & & $10 \cdot 8$ & $\%$ & 0.59 & $0.22,1.58$ \\
\hline Other & 12 & 1.7 & $33 \cdot 3$ & $3 \%$ & $33 \cdot 3$ & & 33.3 & $3 \%$ & 0.49 & $0.12,1.98$ \\
\hline Enrolment location* & & & & & & & & & & \\
\hline FQHC & 300 & $42 \cdot 9$ & 21 & & $46 \cdot 3$ & & $32 \cdot 7$ & $\%$ & 0.58 & $0.21,1.61$ \\
\hline Senior assistance organisations & 194 & $27 \cdot 8$ & $8 \cdot 3$ & & 51 & $\%$ & $40 \cdot 7$ & $\%$ & 1.35 & $0.44,4.13$ \\
\hline Academic primary care clinic & 122 & 17.5 & $18^{\circ}$ & & 48.4 & & 33.6 & $\%$ & 0.72 & $0.24,2.16$ \\
\hline Dept of health & 39 & 5.58 & $25 \cdot 6$ & & $46 \cdot 2$ & & $28 \cdot 2$ & $\%$ & 0.31 & $0.090,1.08$ \\
\hline Other & 44 & $6 \cdot 29$ & $15 \cdot 9$ & & $54 \cdot 6$ & & $29 \cdot 6$ & $\%$ & $\begin{array}{l}\text { Base } \\
\text { category }\end{array}$ & \\
\hline Primary insurance* & & & & & & & & & & \\
\hline Medicare & 153 & $21 \cdot 9$ & $11 \cdot 8$ & & $47 \cdot 1$ & & $41 \cdot 2$ & $\%$ & 0.87 & $0.39,1.93$ \\
\hline Medicare advantage & 137 & $19 \cdot 6$ & $14 \cdot 6$ & & 48.9 & & $36 \cdot 5$ & & 0.59 & $0.26,1.35$ \\
\hline Medicaid & 169 & $24 \cdot 2$ & $19 \cdot 5$ & & 44.4 & & $36 \cdot 1$ & & 1.03 & $0.53,2.01$ \\
\hline Private & 92 & $13 \cdot 2$ & $13 \cdot 0$ & & $50 \cdot 0$ & & $36 \cdot 1$ & & 1.05 & $0.46,2.41$ \\
\hline Other: uninsured, VA, or unlisted & 148 & $21 \cdot 2$ & $23 \cdot 7$ & & $53 \cdot 4$ & & $23 \cdot 0$ & $\%$ & $\begin{array}{l}\text { Base } \\
\text { category }\end{array}$ & \\
\hline Health status and healthcare utilisation & & & & & & & & & & \\
\hline Diabetes diagnosis & 353 & $50 \cdot 5$ & $14 \cdot 2$ & & $51 \cdot 0$ & & $34 \cdot 8$ & $\%$ & 1.42 & $0.87,2.33$ \\
\hline $\begin{array}{l}\text { Average BMI, pre-enrolment } 6 \text { months } \\
\left(\mathrm{kg} / \mathrm{m}^{2}\right) \|\end{array}$ & $33 \cdot 8$ & $9 \cdot 42$ & $32 \cdot 2$ & $10 \cdot 0$ & $33 \cdot 8$ & 9.08 & $34 \cdot 5$ & $9 \cdot 63$ & 1.01 & $0.99,1.04$ \\
\hline $\begin{array}{l}\text { Average systolic blood pressure, } \\
\text { pre-enrolment } 6 \text { months (mmHg) }\end{array}$ & $130 \cdot 5$ & $16 \cdot 5$ & $129 \cdot 9$ & $19 \cdot 3$ & $131 \cdot 0$ & $16 \cdot 0$ & $130 \cdot 2$ & $15 \cdot 7$ & 0.99 & $0.98,1.01$ \\
\hline $\begin{array}{l}\text { Average clinic visits, year } \\
\text { post-enrolment }\end{array}$ & 14.4 & $14 \cdot 2$ & $12 \cdot 2$ & $13 \cdot 1$ & $15 \cdot 1$ & $15 \cdot 6$ & $14 \cdot 6$ & $12 \cdot 5$ & 1.03 & $1.00,1.05 \dagger$ \\
\hline $\begin{array}{l}\text { Average emergency department visits, year } \\
\text { post-enrolment* }\end{array}$ & 1.35 & 3.01 & 1.86 & 3.83 & 1.41 & $3 \cdot 41$ & 1.03 & 1.63 & 0.94 & $0.88,1.01$ \\
\hline $\begin{array}{l}\text { Average hospitalisations, year } \\
\text { post-enrolment }\end{array}$ & 0.49 & $1 \cdot 18$ & 0.53 & $1 \cdot 21$ & 0.54 & 1.27 & 0.40 & 1.04 & 0.81 & $0.75,0.98 \dagger$ \\
\hline
\end{tabular}

${ }^{*}$ Categories with statistically significant ANOVA or $\chi^{2}$ test $(P<0.05)$, indicating difference between Never-Spenders, Sometimes Spenders and Frequent Spenders. tStatistically significant coefficient $(P<0.05)$ for multivariate logistic regression with ever-utilising programme as binary outcome.

$\ddagger$ Continuous variables are presented as Mean (SD), while categorical variables show row percentages (i.e. proportion within each spender type).

$\S n 607$ for multivariate logistic regression.

$\| n 609$.

In 610

${ }^{*}$ Categories with $P<0.01$.

t†Coefficient with $P<0.01$

All other $n 699$ unless specified.

spending ( $\beta=\$ 0.80$ per Prescription Dollar spent or $\$ 8.00$ per 10 Prescription Dollars, $P<0 \cdot 001)$. In both regression models for the full sample, non-fruit and vegetable spending, Hispanic ethnicity and other race were associated with higher fruit and vegetable spending.

Fruit and vegetable expenditure share

Frequent Spender status was associated with higher monthly fruit and vegetable expenditure share $(\beta=3.3 \%, P=0.007)$. The change in expenditure share was $3.9 \%$ for participants with diabetes compared with $2.9 \%$ for those without diabetes. The association was statistically significant for the diabetes subsample, but it did not reach statistical significance for the non-diabetes subsample. Monthly Produce Prescription Dollars spent was also associated with higher expenditure share $(\beta=0.41 \%$ per Prescription Dollar or $4.1 \%$ per 10 Prescription Dollars, $P<0.001)$. In both regression models for the full sample, older age and Hispanic ethnicity were significantly associated with expenditure share. Non-fruit and vegetable spending and white race were associated with lower expenditure share.

\section{Fruit and vegetable variety}

Frequent Spender status was associated with increased fruit and vegetable variety, or the number of unique fruit and vegetable items in a month $(\beta=2.52, P<0.001)$. The 
Table 2 Comparison between individuals with diabetes diagnosis $(n 353)$ and without diabetes diagnosis $(n 346)$ participating in a produce prescription programme, Durham, NC, 2018-2019

\begin{tabular}{|c|c|c|c|c|}
\hline \multirow[b]{2}{*}{ Category } & \multicolumn{2}{|c|}{$\begin{array}{l}\text { Participants without } \\
\text { diabetes diagnosis }\end{array}$} & \multicolumn{2}{|c|}{$\begin{array}{l}\text { Participants with } \\
\text { diabetes diagnosis }\end{array}$} \\
\hline & Mean & $\mathrm{SD}$ & Mean & SD \\
\hline \multirow[t]{2}{*}{ Average age ${ }^{\star}$ (years) } & $55 \cdot 6$ & $15 \cdot 8$ & $61 \cdot 2$ & $11 \cdot 6$ \\
\hline & $n$ & $\%$ & $n$ & $\%$ \\
\hline \multicolumn{5}{|l|}{ Sex } \\
\hline Female & 252 & $72 \cdot 8$ & 254 & $72 \cdot 0$ \\
\hline Male & 94 & $27 \cdot 2$ & 99 & $28 \cdot 0$ \\
\hline \multicolumn{5}{|l|}{ Race or ethnicity } \\
\hline Black & 280 & $80 \cdot 9$ & 289 & 81.9 \\
\hline White non-Hispanic & 39 & 11.3 & 42 & 11.9 \\
\hline Hispanic (regardless of race) & 20 & $5 \cdot 8$ & 17 & 4.8 \\
\hline Other & 7 & $2 \cdot 0$ & 5 & 1.4 \\
\hline \multicolumn{5}{|l|}{ Enrolment location ${ }^{\star *}$} \\
\hline Federally qualified health centre & 172 & $49 \cdot 7$ & 128 & $36 \cdot 3$ \\
\hline Senior assistance organisations & 84 & $24 \cdot 3$ & 110 & $31 \cdot 2$ \\
\hline Academic primary care clinic & 54 & $15 \cdot 6$ & 68 & $19 \cdot 3$ \\
\hline Dept of Health & 15 & $4 \cdot 3$ & 24 & $6 \cdot 8$ \\
\hline Other & 21 & $6 \cdot 1$ & 23 & $6 \cdot 5$ \\
\hline \multicolumn{5}{|l|}{ Primary insurance** } \\
\hline Medicare & 54 & $15 \cdot 6$ & 99 & $28 \cdot 1$ \\
\hline Medicare advantage & 57 & 16.5 & 80 & $22 \cdot 7$ \\
\hline Medicaid & 89 & $25 \cdot 7$ & 80 & $22 \cdot 7$ \\
\hline Private & 51 & $14 \cdot 7$ & 41 & 11.6 \\
\hline \multirow[t]{2}{*}{ Other, uninsured, VA, or unlisted } & 95 & 27.5 & 53 & $15 \cdot 0$ \\
\hline & Mean & SD & Mean & SD \\
\hline \multicolumn{5}{|l|}{ Health status and healthcare utilisation } \\
\hline Average $\mathrm{HbA} 1 \mathrm{c}$ in pre-enrolment 6 months $(\%)^{\star *}, \dagger$ & 5.65 & 0.34 & $8 \cdot 32$ & $2 \cdot 16$ \\
\hline Average BMI in pre-enrolment 6 months $\left(\mathrm{kg} / \mathrm{m}^{2}\right)^{\star \star}, \ddagger$ & $30 \cdot 9$ & $9 \cdot 0$ & $36 \cdot 2$ & $9 \cdot 1$ \\
\hline Average systolic blood pressure, pre-enrolment 6 months $(\mathrm{mmHg})^{\star \star}, \S$ & $128 \cdot 2$ & $16 \cdot 5$ & 132.5 & $16 \cdot 3$ \\
\hline Average clinic visits, year post-enrolment ${ }^{\star *}$ & 11.5 & $12 \cdot 0$ & $17 \cdot 3$ & $15 \cdot 6$ \\
\hline Average emergency department visits, year post-enrolment & $1 \cdot 26$ & $3 \cdot 21$ & 1.44 & $2 \cdot 81$ \\
\hline \multirow[t]{2}{*}{ Average hospitalisations, in year post-enrolment ${ }^{\star *}$} & 0.29 & 0.74 & 0.69 & 1.47 \\
\hline & $n$ & $\%$ & $n$ & $\%$ \\
\hline \multicolumn{5}{|l|}{ Programme utilisation } \\
\hline Never spender & 68 & $19 \cdot 7$ & 50 & $14 \cdot 2$ \\
\hline Sometimes spender & 159 & $46 \cdot 0$ & 180 & $51 \cdot 0$ \\
\hline \multirow[t]{2}{*}{ Frequent spender } & 119 & 34.4 & 123 & $34 \cdot 8$ \\
\hline & Mean & SD & Mean & SD \\
\hline \multicolumn{5}{|l|}{ Baseline healthy food purchasing in month before enrolment } \\
\hline Fruit and vegetable spending (\$) & 8.53 & $19 \cdot 7$ & 7.43 & 16.5 \\
\hline Fruit and vegetable expenditure share (\%)\| & $13 \cdot 0$ & $12 \cdot 8$ & $16 \cdot 2$ & $17 \cdot 8$ \\
\hline Fruit and vegetable diversity (unique items) $\|$ & $7 \cdot 13$ & 8.43 & 5.67 & 6.47 \\
\hline
\end{tabular}

${ }^{*}$ Categories with statistically significant ANOVA or $\chi^{2}$ test $(P<0.05)$, indicating difference between Never-Spenders, Sometimes Spenders and Frequent Spenders.

† $n 61$ and 287 for non-diabetes and diabetes subsamples, respectively.

$\ddagger n 279$ and 330 for non-diabetes and diabetes subsamples, respectively.

$\S n 280$ and 330 for non-diabetes and diabetes subsamples, respectively.

$\| n 154$ and 175 for non-diabetes and diabetes subsamples, respectively.

${ }^{\star *}$ Categories with $P<0.01$.

Continuous variables presented as Mean (SD), while categorical variables show column percentages (i.e. proportion within the subsamples with diabetes and without diabetes).

diversity increase was $2 \cdot 30$ items for participants with diabetes compared with 2.99 items for those without diabetes. Monthly Produce Prescription Dollars spent was also associated with increased variety $(\beta=0.23$ or 2.3 items per 10 Prescription Dollars, $P<0 \cdot 001)$. In the full sample, nonfruit and vegetable spending, older age, Hispanic ethnicity and other race were associated with increased variety.

\section{$\operatorname{Aim} 3$}

Health outcomes for participants with diabetes

For participants with diabetes, there were no statistically significant relationships between higher programme utilisation and diabetes-related outcomes, including BMI $(\beta=0.64, P=0.54)$, HbA1c $(\beta=-0.15, P=0.53)$ and systolic blood pressure $(\beta=-1.09, P=0.51)$.

\section{Discussion}

The current study evaluated a Produce Prescription Programme. Our primary interests were programme utilisation patterns and the programme's effect on healthy food purchasing, but we also assessed diabetes-related health outcomes.

Higher programme utilisation likelihood after enrolment (Aim 1) was associated with being female and older. This is 


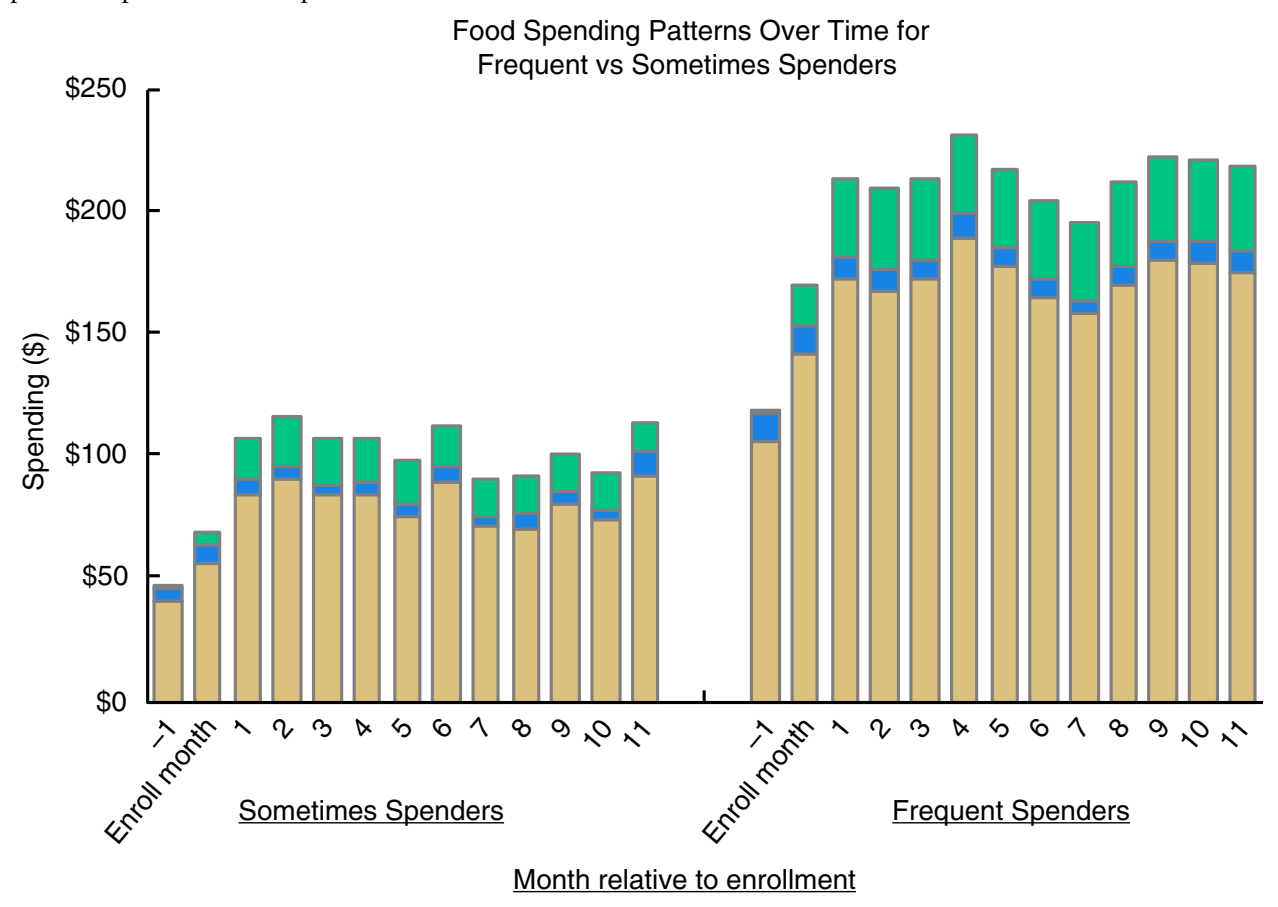

Fig. 2 (colour online) Food purchasing patterns for Produce Prescription Programme participants by utilisation level. Changes in food spending for participants month to month among those who were Frequent or Sometimes Spenders. See Supplemental Material for a table of data included in this figure. Two stacked bar charts showing spending patterns for Produce Prescription Programme Frequent Spenders and Sometimes Spenders. Each stacked bar has three segments to represent fruit and vegetable spending paid with Produce Prescription Dollars, fruit and vegetable spending paid with other methods and non-fruit and vegetable spending. Accompanying data are shown in table form in the Supplemental Material

consistent with women often assuming primary responsibility for household shopping and meal preparation ${ }^{(13,14)}$. That seniors were more likely to utilise the Produce Prescription programme may be consistent with longer eligibility periods for elderly SNAP recipients ${ }^{(15)}$. Elderly SNAP recipients are more likely to remain enrolled in SNAP, though the overall proportion of eligible seniors who enrol in SNAP is lower than those of all other age groups $^{(16)}$. This Produce Prescription Programme requires individuals to maintain SNAP eligibility, so younger individuals may have lost their ability to receive the Produce Prescription due to losing SNAP eligibility. It is also possible that two enrolment sites, which primarily serve seniors, engaged in more tailored outreach that led to higher utilisation.

Hospitalisations were negatively associated with any programme utilisation, and ED visits were negatively associated with more frequent programme utilisation. This is consistent with prior research showing a bidirectional relationship between food insecurity and hospitalisations, particularly in seniors. In one study, elderly individuals experiencing food insecurity were $40 \%$ more likely to be having concurrent hospitalisation. That study found hospitalisation was associated with a $50 \%$ higher chance of becoming food insecure in the future ${ }^{(17)}$. A hospitalisation can be a health and financial shock and a significant barrier to shopping for and cooking healthy food. Differences in programme utilisation by enrolment location suggest that there may have been site-specific variation in screening, communication strategies or instructional materials.

Higher programme utilisation (measured as being a Frequent Spender or higher Produce Prescription Dollars spent) was associated with higher fruit and vegetable spending, expenditure share and variety (Aim 2). This is consistent with other Produce Prescription Programmes, as well as non-clinic nutrition incentive programmes. For instance, a Produce Prescription Programme for patients with hypertension was associated with an increase of 3.3 to 4.9 daily fruit and vegetable servings and decreases in fast food consumption based on self-reporting ${ }^{(18)}$. In another study, 177 patients who received a clinic waiting room intervention to recruit people into the Southeast Michigan Double Up Food Bucks programme reported consuming an average of 0.65 more servings of fruits and vegetables at 3 months ${ }^{(19)}$. For monthly fruit and vegetable spending and variety, the positive effects associated with being a Frequent Spender were smaller for participants with diabetes compared with participants without diabetes. This may be related to the older average age of patients with diabetes in our sample. Older people face additional food access barriers, including living in food deserts and having functional limitations that may make it harder to physically go to a grocery store to utilise the programme ${ }^{(20)}$. This Produce Prescription Programme primarily covered uncooked foods, which could pose 
Table 3 Effects of produce prescription programme utilisation on fruit and vegetable purchasing, Durham, NC, 2018-2019

\begin{tabular}{|c|c|c|c|c|c|c|c|c|c|}
\hline \multirow[b]{2}{*}{ Key variable of interest } & \multicolumn{3}{|c|}{$\begin{array}{l}\text { Effect on fruit and vegetable spending } \\
\qquad(\$)\end{array}$} & \multicolumn{3}{|c|}{$\begin{array}{l}\text { Effect on fruit and vegetable } \\
\text { expenditure share (\%) }\end{array}$} & \multicolumn{3}{|c|}{$\begin{array}{l}\text { Effect on fruit and vegetable diversity } \\
\text { (unique items) } \dagger\end{array}$} \\
\hline & $\begin{array}{l}\text { Full } \\
\text { sample }\end{array}$ & $\begin{array}{l}\text { Diabetes } \\
\text { sample }\end{array}$ & $\begin{array}{l}\text { Non-diabetes } \\
\text { sample }\end{array}$ & $\begin{array}{l}\text { Full } \\
\text { sample }\end{array}$ & $\begin{array}{l}\text { Diabetes } \\
\text { sample }\end{array}$ & $\begin{array}{l}\text { Non-diabetes } \\
\text { samplef }\end{array}$ & $\begin{array}{l}\text { Full } \\
\text { sample }\end{array}$ & $\begin{array}{l}\text { Diabetes } \\
\text { sample }\end{array}$ & $\begin{array}{l}\text { Non-diabetes } \\
\text { sample }\end{array}$ \\
\hline $\begin{array}{l}\text { Frequent spender (relative to } \\
\text { sometimes-spender) }\end{array}$ & $\$ 8 \cdot 77^{\star *}$ & $\$ 7 \cdot 51^{* *}$ & $\$ 10 \cdot 11^{* *}$ & $3.3 \% * *$ & $3.8 \% *$ & $3.2 \%$ & $2 \cdot 52^{* *}$ & $2 \cdot 30^{\star *}$ & $2.99^{* *}$ \\
\hline $\begin{array}{l}\text { Monthly produce prescription } \\
\text { dollars spent (\$) }\end{array}$ & $\$ 0 \cdot 80^{* *}$ & $\$ 0 \cdot 80^{\star *}$ & $\$ 0.79^{\star \star}$ & $0.41 \%$ ** & $0.40 \%$ ** & $0.41 \%$ ** & $0.23^{\star \star}$ & $0.24^{\star *}$ & $0.23^{\star *}$ \\
\hline
\end{tabular}

*Significant at $P<0.05$ level.

** Significant at $P<0.01$ level.

†Fruit and vegetable diversity is defined as the number of unique fruits and vegetables purchased in a month based on number of distinct item barcodes.

†Regression coefficient $P=0.065$.

Full sample regressions include 581 participants; the diabetes subsample has 303 participants and the non-diabetes subsample has 278 participants.

additional burden challenge for older participants with diabetes who may be affected by tremors, neuropathy, arthritis or lack of strength ${ }^{(21)}$.

There were no significant changes in diabetes-related health outcomes after up to 1 year of programme participation (Aim 3). Previous studies have been inconsistent in showing improved health outcomes despite healthier food consumption. A Detroit Produce Prescription Programme that gave 65 participants $\$ 40$ per month for use at a clinic-located farmers market was associated with an average decrease in HbA1c from 9.54 to 8.53 , but there were no changes in BMI or blood pressure ${ }^{(22)}$.

Our Produce Prescription Programme served a community that was disproportionately elderly, Black and low-income. These factors mean a $\$ 40$ per month intervention may not sufficiently address multiple structural inequities such as racism and sexism. Nonetheless, this Produce Prescription Program was associated with increased healthy food purchasing including for participants with diabetes. Moreover, the positive health effects of good diet can take years to materialise. A systematic review found that several diets that emphasise fruits and vegetables are associated with better long-term cardiometabolic outcomes, including Mediterranean, Dietary Approaches to Stop Hypertension, vegetarian, Nordic and Portfolio diets ${ }^{(23)}$. Therefore, we should continue making healthy diets more accessible for people with the most socio-economic challenges.

\section{Strengths and limitations}

The current study demonstrates the feasibility and strength of using two large existing data sets with food transaction and health data to measure Produce Prescription Programme impact on food purchasing and clinical health measures. Our study goes beyond prior Produce Prescription Programme research that has mostly relied on self-reported food security and survey-based food intake assessments.

Though we controlled for measured and unobserved participant differences through panel regression, participants were not randomised. For some participants, it was not possible to match all their health and food transaction data, possibly due to those participants being recruited from settings that are not part of Durham's integrated EHR system (which covers the vast majority of those recruited at federally qualified health centre and academic clinic settings but not necessarily the participants enrolled through community organisations). This limits our ability to make strong claims about programme efficacy and causality. On the other hand, the nonrandomised design enabled assessment of utilisation predictors (Aim 1) to inform programme implementation.

The study was limited by imprecise food consumption measures. We only had access to transaction data from our partner grocery store chain, but not from other grocery stores, convenience stores, markets or other food assistance programmes. We lacked qualitative data on food preparation, consumption and/or waste. We also could not completely measure substitution and income effects due to programme participation. We expect a substitution effect towards more fruits and vegetables because they are effectively cheaper, but the income effect may increase total food consumption. In our study, we note total food spending increased, which means there is a theoretical possibility of not only more healthy food consumption but also more unhealthy food consumption. The dietary significance of competing increases in healthy and unhealthy purchases is difficult to quantify.

Due in part to programme features intended to minimise clinician burden, healthcare providers did not systematically ask patients about self-reported food security, as has been done in other studies. A study of Wholesome Wave's multi-city Produce Prescription Programme was associated with improved food security score among 578 low-income households ${ }^{(24)}$. Also to minimise clinician burden, the Produce Prescription enrollers did not collect household size data, which prevents converting purchase data into food servings. However, we controlled indirectly for household size by including non-fruit and vegetable spending as a covariate in our food purchasing regressions. 
Nonetheless, our study's strength lies in assessing real-world changes in participant food purchasing. This intervention likely increased food security, as indicated by the increases in nutritious food purchases and dietary diversity. However, without data on individual food intake or self-reported food security, we must be cautious about drawing strong conclusions about food quantity sufficiency.

This Produce Prescription Programme reached numerous people of colour and of lower socio-economic status, particularly by enrolling through a federally qualified health centre. While using SNAP for programme delivery enhances its efficiency and ease of use, this may have excluded Hispanic or Latino individuals, particularly those who are undocumented or have concerns about receiving government-administered assistance. This may partly explain the relatively low number of Hispanic participants in our sample. We found that despite there being fewer Hispanic or Latino Frequent Spenders, those who were Frequent Spenders actually had higher fruit and vegetable expenditures than other ethnic groups. However, we chose not to make strong conclusions about the effects of Hispanic ethnicity due to the relatively small number in our sample.

It is worth replicating and expanding this work: with larger samples, more healthcare settings (e.g. including paediatrics clinics), longer programme duration, analyses of different food groups including healthy and unhealthy foods and beverages and higher subsidy amounts to achieve clinically significant improvements in chronic disease management. Future Produce Prescription Programmes should include targeted outreach and potentially programme delivery without reliance on SNAP if it does not sacrifice efficiency. These efforts would enable serving a higher proportion of young and/or Hispanic people. For serving older individuals who may have additional co-morbidities and functional limitations, Produce Prescription Programmes should consider modalities involving home grocery delivery, online grocery shopping using SNAP and/or additional emphasis on healthy prepared foods - all of which have become more widespread as part of the retail food environment and food security interventions during the COVID-19 pandemic $^{(25,26)}$. Following participants for a longer period may provide insight on long-term cardiometabolic outcomes. Future studies should also collect qualitative food consumption and food security data. It is also of interest to assess how Produce Prescription Programmes affect provider-patient relationships. For example, Produce Prescription Programmes have been associated with more frequent food and nutrition conversations with healthcare providers ${ }^{(18)}$. There is also a continued need for implementation research to improve healthcare provider and community organisation workflow.

\section{Implications for nutrition and public bealth}

Produce Prescription Programmes can increase healthy food purchasing by bringing together clinics, community organisations and food retailers in novel ways. They are one of several forms of delivering 'food as medicine.' Food as medicine interventions connect health systems and food systems, including local food businesses ${ }^{(27)}$.

For healthcare providers, Produce Prescription Programmes provide a way to elevate the importance of healthy eating, raise awareness of local resources and further patient education ${ }^{(28)}$. For healthcare systems, Produce Prescription Programmes can improve diet-related illness management. Food as medicine interventions can decrease healthcare costs based on studies showing decreased health expenditures for those who receive SNAP and home meal delivery following hospitalisation ${ }^{(29,30)}$. Though it is difficult to determine causal direction, our results show a correlation between higher Produce Prescription Programme utilisation and fewer hospitalisations, and therefore, possibly healthcare cost reduction. Adding to evidence that Produce Prescription Programmes may reduce healthcare costs, a microsimulation study about Medicare and Medicaid beneficiaries estimated that a fruit and vegetable incentive could prevent 1.93 million CVD events and save $\$ 39.7$ billion in healthcare costs ${ }^{(31)}$. Given the potential for health improvement and cost savings, health systems and insurance entities should consider making Produce Prescription Programmes medically reimbursable alongside other social drivers of health as part of the transition to value-based care ${ }^{(32)}$.

The current study reinforces the business case for grocery retailers to adopt Produce Prescription Programmes because of evidence that they expanded customer loyalty among programme participants, similar to other nutrition incentive programmes ${ }^{(7)}$. Additional analyses not elaborated upon in this paper showed that across all participants, total food spending at our partner grocery store chain grew after enrolment. This suggests that Produce Prescription Programmes may be associated with more regular shopping at the partner food retailer.

Our study shows how healthy food complements clinical care, medication, physical activity and other lifestyle changes. Produce Prescription Programmes make healthy food a major part of a patient's health plan, yet their delivery through SNAP and grocery store loyalty programmes integrates participation seamlessly into the shopping experience. Produce Prescription Programmes can increase patient capacity to follow healthcare providers' dietary advice with dignity and autonomy. Ultimately, food as medicine can enable health and food systems to deliver integrated services that improve healthy food access and chronic disease prevention and management. 


\section{Acknowledgements}

Acknowledgements: The authors thank Reinvestment Partners for designing and implementing this Produce Prescription Programme and the healthcare workers who enrolled their patients. Thanks to Shu Wen Ng and her team at UNC Chapel Hill for lending their expertise in food transaction data categorisation. The authors would also like to thank Chris Everett, Eang King and Corinna Sorenson for research and logistical support. We would like to express gratitude to Elizabeth Onstwedder and Adelaide Xie for feedback and revisions. Data analysis was performed on STATA 16 and Microsoft Excel. The graphical abstract includes the following images: 'clinic' by roughen.line, 'Shopping Cart' by Arthur Shlain and 'fruits and vegetables' by Tatina Vazest from the Noun Project. Financial support: The Produce Prescription Programme evaluated in the current study was funded by the US Department of Agriculture Food Insecurity Nutrition Incentive programme, and the current study was funded by Blue Cross Blue Shield of North Carolina Foundation. Conflicts of interest: The Produce Prescription Programme evaluated in the current study was funded by the US Department of Agriculture Food Insecurity Nutrition Incentive programme, and the current study was funded by Blue Cross Blue Shield of North Carolina Foundation. The funders did not play a role in design or conduct of the study; collection, analysis or interpretation of the data or manuscript preparation, review or approval. Authorship: J.X. contributed to programme implementation, hypothesis creation, data cleaning, statistical modelling, literature analysis, manuscript writing and table/figure creation. A.P. contributed to research design, including institutional review board protocols and writing. N.C. was responsible for programme design, fundraising, administration, implementation in collaboration with the grocery store chain, data access facilitation and manuscript editing. T. $\varnothing$. provided guidance on research design, statistical modelling and manuscript writing. Ethics of buman subject participation: The current study was conducted according to the guidelines laid down in the Declaration of Helsinki, and all procedures involving research study participants were approved by Duke Health Institutional Review Board. A waiver of consent was requested and deemed acceptable by the Duke Health Institutional Review Board because of the research team's secondary data analysis used of existing programme administrative data (including food transaction data) and retrospective electronic health record data collected as standard of care. All data already collected by programme implementers and healthcare teams were part of the standard of care with no additional data being collected beyond what patients experience during their clinic visits and part of participation in the programme. Clinics and enrollers indicated that they offered programme enrolment to as many eligible patients as they could and communicated to patients that participation was voluntary. All efforts to maximise patient privacy were made with all analysis and data transfer occurring in secure platforms.

\section{Supplementary material}

For supplementary material accompanying this paper visit https://doi.org/10.1017/S1368980021001828

\section{References}

1. Cooksey-Stowers K, Schwartz MB \& Brownell KD (2017) Food swamps predict obesity rates better than food deserts in the United States. Int JEnviron Res Public Health 14, 1366.

2. Coleman-Jensen A, Rabbitt MP \& Gregory CA (2019) Food Security Status of U.S. Households in 2018. US Department of Agriculture Economic Research Service. https://www. ers.usda.gov/topics/food-nutrition-assistance/food-securityin-the-us/key-statistics-graphics.aspx\#foodsecure.

3. (2019) Household Food Insecurity Rates by State, 2016-2018. Food Research and Action Center. https://frac.org/maps/ food-security/tables/tab1_foodinsecurity_2016_2018.html.

4. Mortiboy M (2021) Durham County Community Health Assessment 2020. Durham: Department of Public Health. https://healthydurham.org/cms/wp-content/uploads/2021/ 03/2020-CHA-Final-Draft-1.pdf.

5. Andreyeva T, Tripp AS \& Schwartz MB (2015) Dietary quality of Americans by supplemental nutrition assistance program participation status: a systematic review. Am J Prev Med 49, 594-604.

6. Berkowitz SA, Karter AJ, Corbie-Smith G et al. (2018) Food insecurity, food 'deserts,' and glycemic control in patients with diabetes: a longitudinal analysis. Diabetes Care 41, 1188-1195.

7. Parks CA, Stern KL, Fricke HE et al. (2020) Healthy food incentive programs: findings from food insecurity nutrition incentive programs across the United States. Health Promot Pract 21, 421-429.

8. (2018) Healthy Food Playbook: Fruit and vegetable incentives. Health Care Without Harm. https://foodcommunity benefit.noharm.org/resources/implementation-strategy/ program-fruit-and-vegetable-incentives.

9. Swartz H (2018) Produce Rx programs for diet-based chronic disease prevention. AMA J Ethics 20, 960-973.

10. Yoder AD, Proaño GV \& Handu D (2020) Retail nutrition programs and outcomes: an evidence analysis center scoping review. J Acad Nutr Diet. Published online: 20 November 2020. doi: 10.1016/j.jand.2020.08.080.

11. Makelarski JA, Abramsohn E, Benjamin JH et al. (2017) Diagnostic accuracy of two food insecurity screeners recommended for use in health care settings. Am J Public Health 107, 1812-1817.

12. Steele-Adjognon M \& Weatherspoon D (2017) Double up food bucks program effects on SNAP recipients' fruit and vegetable purchases. BMC Public Health 17, 946.

13. Schaeffer K (2019) Among U.S. couples, women do more cooking and grocery shopping than men. Pew Res Cent. https://www.pewresearch.org/fact-tank/2019/09/24/amongu-s-couples-women-do-more-cooking-and-grocery-shoppingthan-men/.

14. Lee Anne F, Bisakha S, Kilgore ML et al. (2014) The influence of gender, age, education, and household size on meal preparation and food shopping responsibilities. Public Health Nutr 17, 2061-2070.

15. Gregory M, Tracy V, Heather K et al. (2014) Understanding the Rates, Causes, and Costs of Churning in the Supplemental Nutrition Assistance Program (SNAP). USDA 
Food and Nutrition Service and Urban Institute. https:// www.fns.usda.gov/snap/understanding-rates-causes-andcosts-churning-supplemental-nutrition-assistance-programsnap.

16. Alma V (2019) Trends in Supplemental Nutrition Assistance Program Participation Rates: Fiscal Year 2010 to Fiscal Year 2017. USDA Food and Nutrition Service. https://www.fns. usda.gov/snap/trends-supplemental-nutrition-assistanceprogram-participation-rates-fiscal-year-2010.

17. Bergmans RS, Mezuk B \& Zivin K (2019) Food insecurity and geriatric hospitalization. Int J Environ Res Public Health 16, 2294.

18. Trapl ES, Smith S, Joshi K et al. (2018) Dietary impact of produce prescriptions for patients with hypertension. Prev Chronic Dis 15, E138.

19. Cohen AJ, Richardson CR, Heisler M et al. (2017) Increasing use of a healthy food incentive: a waiting room intervention among low-income patients. Am J Prev Med 52, 154-162.

20. Petersen CL, Brooks JM, Titus AJ et al. (2019) Relationship between food insecurity and functional limitations in older adults from 2005-2014 NHANES. J Nutr Gerontol Geriatr 38, 231-246.

21. Stanley K (2014) Nutrition considerations for the growing population of older adults with diabetes. Diabetes Spectr Publ Am Diabetes Assoc 27, 29-36.

22. Bryce R, Guajardo C, Ilarraza D et al. (2017) Participation in a farmers' market fruit and vegetable prescription program at a federally qualified health center improves hemoglobin A1C in low income uncontrolled diabetics. Prev Med Rep 7, 176-179.

23. Kahleova H, Salas-Salvadó J, Rahelić D et al. (2019) Dietary patterns and cardiometabolic outcomes in diabetes: a summary of systematic reviews and meta-analyses. Nutrients 11, 2209.
24. Ridberg RA, Bell JF, Merritt KE et al. (2019) A pediatric fruit and vegetable prescription program increases food security in low-income households. J Nutr Educ Behav $\mathbf{5 1}$, 224.e1-230.e1.

25. Leone LA, Fleischhacker S, Anderson-Steeves B et al. (2020) Healthy food retail during the COVID-19 pandemic: challenges and future directions. Int J Environ Res Public Health 17, 7397.

26. (2021) FNS Launches the Online Purchasing Pilot. USDA Food Nutr Serv. https://www.fns.usda.gov/snap/onlinepurchasing-pilot.

27. Jaacks LM \& Bellows AL (2017) Let food be thy medicine: linking local food and health systems to address the full spectrum of malnutrition in low-income and middle-income countries. BMJ Glob Health 2, e000564.

28. Goddu AP, Roberson TS, Raffel KE et al. (2015) Food Rx: a community-university partnership to prescribe healthy eating on the south side of Chicago. J Prev Interv Community 43, 148-162.

29. Berkowitz SA, Seligman HK, Rigdon J et al. (2017) Supplemental nutrition assistance program (SNAP) participation and health care expenditures among low-income adults. JAMA Intern Med 177, 1642-1649.

30. Berkowitz SA, Terranova J, Hill C et al. (2018) Meal delivery programs reduce the use of costly health care in dually eligible Medicare and Medicaid beneficiaries. Health Aff (Millwood) 37, 535-542.

31. Lee Y, Mozaffarian D, Sy S et al. (2019) Cost-effectiveness of financial incentives for improving diet and health through Medicare and Medicaid: a Microsimulation study. PLOS Med 16, e1002761.

32. Lundeen EA (2017) Clinical-community partnerships to identify patients with food insecurity and address food needs. Prev Chronic Dis 14, E113. 